\title{
ON A COMMUTATIVE EXTENSION OF A BANACH ALGEBRA ${ }^{1}$
}

\author{
F. T. BIRTEL
}

Introduction. Let $A$ be a commutative semi-simple Banach algebra and let $\Delta(A)$ be the set of nonzero multiplicative functionals on $A$. Denote by $A^{\prime}$ the strongly closed span of $\Delta(A)$ and by $A^{\prime \prime}$ the Banach space adjoint of $A^{\prime}$. Modifying a construction of $\mathrm{R}$. Arens [1] we introduce a multiplication in $A^{\prime \prime}$ under which $A^{\prime \prime}$ becomes a commutative Banach algebra. $A$ is algebraically isomorphic to a subalgebra of $A^{\prime \prime}$ and the isomorphism is continuous. Indeed, we will henceforth need that the embedding of $A$ in $A^{\prime \prime}$ be topological. If $A$ has a weak bounded approximate identity, then the algebra $A^{m}$ of multipliers of $A$ (see $[3 ; 4]$ ) is likewise embeddable in $A^{\prime \prime}$ and the isomorphism is again continuous. In this paper we are concerned with identification of $A^{m}$ in $A^{\prime \prime}$. For example, if $A$ is, in addition to the above assumptions, regular and Tauberian and if $\Delta(A)$ is discrete, then $A^{m}$ and $A^{\prime \prime}$ are topologically and algebraically isomorphic. The main result is the following: For $A$ with approximate identity in $j_{A}(\infty)$, an element $F$ of $A^{\prime \prime}$ is a multiplier of $A$ if and only if $F$ belongs locally to $\hat{A}$ at each point of $\Delta(A)$.

The multiplier algebra of the group algebra $L_{1}(G)$ of a locally compact abelian group $G$ is the algebra $M(G)$ of bounded measures on $G$. In this case, our main theorem closely parallels Eberlein's necessary and sufficient condition for a function to be a Fourier-Stieltjes transform of a measure on $G$. See [5]. As an application, we construct $A^{\prime \prime}$ and use Eberlein's theorem to determine the algebra of multipliers of the $L_{1}$-algebra of certain semi-groups $G_{+}$.

The author wishes to thank Professor I. Glicksberg for calling his attention to $A^{\prime \prime}$ and for many helpful suggestions.

1. Preliminaries. $\hat{A}$ is the function algebra on $\Delta(A)$ isomorphic to the commutative semi-simple Banach algebra $A$ in the Gelfand theory. Let $A^{m}$ be the set of all complex-valued functions on $\Delta(A)$ such that $f \cdot \hat{x} \in \hat{A}$ for all $x \in A$. Each $f \in A^{m}$ determines a bounded operator $f$ on $A$ given by $(f x)^{\wedge}=f \hat{x}$. The set of all such operators with the uniform operator norm is a commutative semi-simple Banach algebra under the obvious operations. It is called the algebra of multipliers

Presented to the Society, January 23, 1961, under the title On the adjoint of the closed span of multiplicative functionals; received by the editors September 21, 1961.

1 This paper was prepared in part while the author held an Office of Naval Research Postdoctoral Associateship at Yale University. 
of $A$ and is designated by $A^{m}$. (The ambiguous use of $A^{m}$ will be clear in context.) $\Delta(A)$ is homeomorphic to a subset of $\Delta\left(A^{m}\right)$ both in their weak* topologies. See [3].

$A$ is said to be regular if functions in $\hat{A}$ separate points and closed sets of $\Delta(A), j_{A}(\infty)$ is the set of $x \in A$ such that $\hat{x}$ has compact support. Regular $A$ is called Tauberian, if $j_{A}(\infty)$ is dense in $A$.

$A^{*}$ will denote the Banach space adjoint of $A$ and $A^{* *}$, the second adjoint. If $f$ is a function on $X$ and $Y \subset X, f \mid Y$ will denote the restriction of $f$ to $\mathrm{Y}$. Let $K$ be the complex number field.

2. Construction of $A^{\prime \prime}$. For completeness, we first sketch the construction of $\mathrm{R}$. Arens [1] used to introduce a multiplication in $A^{* *}$.

Define $f x\left(x \in A, f \in A^{*}\right)$ by $f x(y)=f(x y)$ for $y \in A$, define Ff $\left(f \in A^{*}, \quad F \in A^{* *}\right)$ by $F f(x)=F(f x)$ for $x \in A$, define $G F\left(F, G \in A^{* *}\right)$ by $G F(f)=G(F f)$ for $f \in A^{*} . f x$ is linear on $A$ and $|f x(y)|=|f(x y)| \leqq\|f\|\|x y\| \leqq\|f\|\|x\|\|y\|$, hence $f x \in A^{*}$ and $\|f x\|$ $\leqq\|f\|\|x\|$. Similarly, $F f$ is linear on $A$ and $|F f(x)|=|F(f x)| \leqq\|F\|\|f x\|$ $\leqq\|F\|\|f\|\|x\|$, so $F f \in A^{*}$ and $\|F f\| \leqq\|F\|\|f\|$. Also $G F$ is linear on $A^{*}$, $|G F(f)|=|G(F f)| \leqq\|G\|\|F f\| \leqq\|G\|\|F\|\|f\|$, therefore, $G F \in A^{* *}$ and $\|G F\| \leqq\|G\|\|F\|$. As Arens points out, multiplication so defined in $A^{* *}$ is not in general commutative.

In the above construction replace $A^{*}$ by the strongly closed span $A^{\prime}$ of $\Delta(A)$. Indeed, we might even replace $A^{*}$ by the strongly closed span of the Silov boundary $\partial \Delta(A)$ of $\Delta(A) .(\partial \Delta(A)$ is the minimal closed set on which each function $|\hat{x}| \in|\hat{A}|$ maximizes.) We then introduce as in the preceding paragraph a multiplication in $A^{\prime \prime}=A^{\prime *}$ which is defined on a dense subset of $A^{\prime}$ as follows (all sums are finite):

(i) $\left(\sum a_{\nu} p_{\nu}\right) x=\sum a_{\nu} p_{\nu}(x) p_{\nu}$

(ii) $F\left(\sum a_{v} p_{v}\right)=\sum a_{v} F\left(p_{v}\right) p_{\text {, }}$

(iii) $F G$ is given by $F G\left(\sum a_{\nu} p_{v}\right)=\sum a_{v} F\left(p_{v}\right) G\left(p_{v}\right)$

$$
\left(p_{v} \in \Delta(A), x \in A, a_{v} \in K\right)
$$
$\left(F \in A^{\prime \prime}, \sum a_{\nu} p_{\nu} \in A^{\prime}\right)$, $\left(F, G \in A^{\prime \prime}, \sum a_{\nu} p_{\nu} \in A^{\prime}\right)$

$F G$ clearly extends to a function on all of $A^{\prime}$. Multiplication so defined is commutative and exactly as in the Arens construction $\|F G\| \leqq\|F\|\|G\|$.

3. Embeddings. In a natural fashion $A$ can be embedded in $A^{\prime \prime}$ and the embedding is continuous. Let $x^{* *}$ be the element of $A^{* *}$ associated, under the canonical embedding of $A$ in $A^{* *}$, with $x \in A$. Then $x^{* *} \mid A^{\prime} \in A^{\prime \prime}$. And

$$
\left\langle\sum a_{\nu} p_{v},\left(x^{* *} \mid A^{\prime}\right)\left(y^{* *} \mid A^{\prime}\right)\right\rangle=\sum a_{\nu}\left(x^{* *} \mid A^{\prime}\right)\left(p_{v}\right)\left(y^{* *} \mid A^{\prime}\right)\left(p_{\nu}\right)
$$

which agrees with the multiplication in $A^{\prime \prime}$ given by (iii) above. Observe that $x^{* *} \mid \Delta(A)=\hat{x}$. 
In order to realize $A^{m}$ as a subalgebra of $A^{\prime \prime}$, we assume that $A$ has a weak bounded approximate identity; that is, there exists a net $\left\{x_{\delta}\right\}$ in $A$ such that for each finite set $\left\{p_{1}, p_{2}, \cdots, p_{n}\right\} \subset \Delta(A)$, $\lim _{\delta} x_{\delta}\left(p_{\nu}\right)=1$ for $\nu=1,2,3, \cdots, n$ and $\left\{x_{\delta}\right\}$ is strongly bounded. Then

$$
\begin{aligned}
\left|F \sum a_{\nu} p_{\nu}\right| & =\left|\sum a_{\nu} F\left(p_{\nu}\right)\right|=\lim _{\delta}\left|\sum a_{\nu} F \hat{x}_{\delta}\left(p_{\nu}\right)\right| \\
& =\lim _{\delta}\left|F \hat{x}_{\delta} \sum a_{\nu} p_{\nu}\right| \leqq \lim _{\delta}\left\|F x_{\delta}\right\|\left\|\sum a_{\nu} p_{\nu}\right\| \\
& \leqq k\|F\|\left\|\sum a_{\nu} p_{\nu}\right\|
\end{aligned}
$$

for each $F \in A^{m}$ where $\left\|x_{\delta}\right\| \leqq k$ for all $\delta$. (1) implies that $A^{m}$ is embedded continuously in $A^{\prime \prime}$. Semi-simplicity of $A^{m}$ assures that the embedding is one-one. Existence of an approximate identity in the strong sense-a bounded net $\left\{x_{8}\right\} \subset A$ such that $\left\|x_{8} x-x\right\| \rightarrow 0$ for all $x \in A$-evidently suffices to accomplish this embedding.

4. Example. We note the form of $A^{\prime \prime}$ when $A=L_{1}(G)$ with $G$ a locally compact Abelian group: $A^{\prime}$ is the strongly closed span of $\hat{G}$, the dual of $G$. $\hat{G}$ is contained in the essential bounded functions $L_{\infty}(G)$ on $G$. So the norm in this case is the essential supremum norm of $L_{\infty}(G)$. It is well know that the uniform closure of the characters of $G$ are precisely the almost periodic functions on $G$, which in turn can be identified as the continuous functions on the almost periodic compactification $G^{*}$ of $G$. Denote this sup-normed Banach algebra by $C\left(G^{*}\right)$. The adjoint of $C\left(G^{*}\right)$ is $M\left(G^{*}\right) . M(G)$ is isometrically isomorphic to a subset of $M\left(G^{*}\right)$. The multipliers of $L_{1}(G)$ (viz. $M(G)$ ) are precisely the measures of $M\left(G^{*}\right)$ which have weak* continuous restrictions to $\hat{G}$. This last result is due to Eberlein [5]. Also see Glicksberg [6].

TheOREM. Let $A$ be a regular Tauberian commutative semi-simple Banach algebra with discrete maximal regular ideal space $\Delta$. If $A$ is topologically embedded in $A^{\prime \prime}$ and $A^{m}$ is embeddable in $A^{\prime \prime}$, then $A^{m}$ $=A^{\prime \prime}$.

Proof. Let $J=\left\{x \in A: F \hat{x} \in \hat{A}\right.$ where $\left.F \in A^{\prime \prime}\right\}$. Since $A$ is regular and $\Delta$ is discrete,

$$
I=\left\{x_{p}: \hat{x}_{p}(p)=1, \hat{x}_{p}\left(p^{\prime}\right)=0 \text { for } p \neq p^{\prime} ; p, p^{\prime} \in \Delta\right\} \subset J .
$$

Indeed, span $(I) \subset J$. Consequently, $F \cdot j_{A}(\infty) \wedge \subset \hat{A}$. But $x_{n} \rightarrow x$ in $A$, $x_{n} \in j_{A}(\infty)$, implies $F x \in A$, since $F x_{n} \rightarrow F x$ in $A^{\prime \prime}$ and $A$ is embedded topologically in $A^{\prime \prime}$. So $F \cdot j_{A}(\infty)^{-\wedge} \subset \hat{A}$. Therefore $A^{\prime \prime}=A^{m}$.

REMARK. However, even when $A$ is regular and topologically em- 
bedded in $A^{\prime \prime}$, and when $A^{m}$ is embeddable in $A^{\prime \prime}$, it is not true that $A^{m}$ is the set of those elements of $A^{\prime \prime}$ whose restrictions to $\Delta$ are weak* continuous, as one might hope from the previous example and theorem. Specifically, if $D$ is the algebra of complex-valued functions on $[0,1]$ possessing continuous first derivatives, functionals in $A^{\prime \prime}$ can be identified as indefinite integrals of functions in $L_{\infty}[0,1]$, whereas $D^{m}=\hat{D}$, the set of continuously differentiable functions on $[0,1]$.

5. Main theorem. Under certain restrictions, however, we are able to obtain a necessary and sufficient condition that a functional in $A^{\prime \prime}$ actually be a multiplier of $A$.

Definition. Let $A$ be a commutative semi-simple Banach algebra with regular maximal ideal space $\Delta$. A function $f$ on $\Delta$ is said to belong locally to $\hat{A}$ at $p \in \Delta$ if there exists a neighborhood $V$ of $p$ and a function $\hat{x} \in \hat{A}$ such that $f|V=\hat{x}| V$. $f$ belongs locally to $\hat{A}$ at $\infty$ if there exists a neighborhood $W$ of $\infty$ in $\Delta \cup\{\infty\}$ and a function $\hat{x} \in \hat{A}$ such that $f|W=\hat{x}| W$.

In the lemmas which follow we assume that $A$ is a regular commutative semi-simple Banach algebra, that $A$ is embedded topologically in $A^{\prime \prime}$ and that $A^{m}$ is embeddable in $A^{\prime \prime}$.

Lemma 1. If $f \in A^{m}$, then $f$ belongs locally to $\hat{A}$ at each $p \in \Delta$.

Proof. Let $p \in \Delta$. Choose a compact neighborhood $V$ and a function $\hat{x} \in \hat{A}$ such that $\hat{x}(p)=1$ for $p \in V$ (possible, by regularity). Then, for $f \in A^{m}, f \hat{x} \in \hat{A}$ and $f \hat{x}|V=f| V$.

Our next lemma is essentially established in $[7$, p. 85].

LEMMA 2. If a function fon $\Delta$ belongs locally to $\hat{A}$ at each $p \in \Delta \cup\{\infty\}$, then $f \in \hat{A}$.

Proof. If $x_{i} \in A$ and $f=\hat{x}_{i}$ on $U_{i}(i=1,2)$ and if $C$ is a compact subset of $U_{2}$, then there exists $y \in A$ such that $f=y$ on $U_{1} \cup C$ : Using regularity, let $e \in A$ be such that $\hat{e}=1$ on $C$ and $\hat{e}=0$ on $\Delta-U_{2}$. Define $y=x_{2} e+x_{1}(1-e), \hat{y}=\hat{x}_{2}=f$ on $C, \hat{y}=\hat{x}_{1}=f$ on $U_{1}-U_{2}$, and $\hat{y}=f \hat{e}+f(1-\hat{e})=f$ on $U_{1} \cap U_{2}$. Now cover $\Delta \cup\{\infty\}$ with finitely many open sets $U_{1}, U_{2}, \cdots, U_{n}$ such that $f=\hat{x}_{i}$ on $U_{i}$ and $x_{i} \in A$ $(i=1,2, \cdots, n)$. We can find open sets $V_{1}, V_{2}, \cdots, V_{n}$ also covering $\Delta \cup\{\infty\}$ such that $V_{i} \subset \bar{V}_{i} \subset U_{i}(i=1,2, \cdots, n)$. Using the first assertion of the proof and iterating, we have $f=y$ on $\Delta$ with $y \in A$.

If $F \in A^{\prime \prime}$ and if for each $p \in \Delta \cup\{\infty\}$, there is a neighborhood $V$ of $p$ with $F \hat{A}|V \subset \hat{A}| V$, then $F \in A^{m}$, since $F \hat{x}(x \in A)$ belongs locally to $\hat{A}$ at each $p \in \Delta \cup\{\infty\}$ and the previous lemma applies. 
Lemma 3. If $x \in\left(x j_{A}(\infty)^{-}\right)$for every $x \in A$ and if $F \in A^{\prime \prime}$ belongs locally to $\hat{A}$ at each $p \in \Delta$, then $F \in A^{m}$.

Proof. Choose $\left\{x_{n}\right\} \in j_{A}(\infty)$ with the property that $x_{n} x \rightarrow x$ in $A$. Clearly, $F \hat{x}_{n}$ belongs locally to $\hat{A}$ at each $p \in \Delta \cup\{\infty\}$, hence $F \hat{x}_{n} \in \hat{A}$. For any $\epsilon>0$,

$$
\left\|F x_{n}-F x\right\| \leqq\|F\|\left\|x_{n} x-x\right\|<\epsilon \quad \text { for large } n .
$$

And by the topological embedding of $A$ in $A^{\prime \prime}$ we have that $F x \in A$. Therefore $F \in A^{m}$.

The existence of an approximate identity $\left\{u_{\delta}\right\} \subset j_{A}(\infty)$ suffices for $x$ to be in $x j_{A}(\infty)^{-}$and for $A^{m}$ to be embeddable in $A^{\prime \prime}$.

TheOREM. Let $A$ be a regular commutative semi-simple Banach algebra with an approximate identity $\left\{u_{\delta}\right\} \subset j_{A}(\infty)$ and such that $A$ is topologically embeddable in $A^{\prime \prime}$. Then $F \in A^{\prime \prime}$ is a multiplier of $A$ if and only if $F$ belongs locally to $\hat{A}$ at each point of $\Delta$.

The striking similarity of the above theorem with Eberlein's characterization of Fourier-Stieltjes transforms of measures [5] can be emphasized by reformulating the theorem without specific reference to $A^{\prime \prime}$. That $F$ be in $A^{\prime \prime}$ simply requires: there exist a constant $k$ such that

$$
\left|\sum a_{\nu} F\left(p_{\nu}\right)\right| \leqq k\left\|\sum a_{\nu} p_{\nu}\right\|
$$

for all $p_{1}, p_{2}, \cdots, p_{n} \in \Delta$ and complex numbers $a_{1}, a_{2}, \cdots, a_{n}$. Eberlein's proof can be viewed as a verification of the fact that continuous functions on $\Delta=G^{\wedge}$ satisfying (2) belong locally to $\hat{A}=L_{1}(G)^{\wedge}$ and hence are in $A^{m}=M(G)$.

However, it seems worthwhile to give a direct proof $^{2}$ that (2) implies $F$ belongs locally to $L_{1}(G)^{\wedge}$. By regularity, if $D$ is a compact neighborhood of some point of $G^{\wedge}$ and $E$ is an open subset containing $D$, then there exists an $f \in L_{1}(G)$ such that $\hat{f}(p)=1$ for $p \in D$ and $\hat{f}(p)=0$ for $p \in \hat{G}-E$. Consequently, $H=F \cdot \hat{f}$ is in $L_{1} \cap L_{2}(G)$. Let $T^{*}$ denote the inverse Fourier transform. To have $F$ belong locally to $L_{1}(G)^{\wedge}$ it is sufficient to show that $T^{*} H \in L_{2}(G)$ is also in $L_{1}(G)$. Let $U$ be an element in $C(\hat{G})$ of an approximate identity on $\hat{G}$ with 0 having compact support. Then by the Plancherel theorem,

$$
\left|\int_{G} \sum a_{\nu} p_{\nu}(x) T^{*} H(x) \hat{U}(x) d x\right|=\left|\int_{G^{\wedge}}\left(\sum a_{\nu} L_{p_{\nu}} H\right)(p) U(p) d p\right|
$$

Added on May 25, 1962. This proof is due essentially to Professor I. Glicksberg, who provided corrections needed to circumvent certain errors in the author's original argument. 
where $L_{p_{p}}$ denotes left translation by $p_{\nu}^{-1}$. Furthermore,

$$
\sup _{\nu \in \hat{\theta}}\left|\sum a_{\nu} L_{p_{\nu}} H(p)\right| \leqq k\left\|\sum a_{\nu} p_{\nu}\right\|_{\infty}
$$

by (2). Let the support of 0 be $S . S$ is compact in the almost periodic compactification $G^{*}$ of $G$, so by Tietze's theorem we can find an almost periodic function $h \in C\left(G^{*}\right)$ such that

$$
h \mid S=\exp \left(-i \arg \left(T^{*} H\right) \cdot \hat{U}\right)
$$

with $\|h\|_{\infty} \leqq 1$. Then $h$ can be uniformly approximated on $G^{*}$ by a trigonometric polynomial $\sum a, p$, of modulus less than $1+\epsilon$. Using this trigonometric polynomial in (3) and (4) we have

$$
\int_{G}\left|\left(T^{*} B\right) 0\right| \leqq\left|\int_{\sigma}\left(T^{*} H\right) \cdot \sum a_{\nu} p_{\nu} \cdot 0\right| \leqq M
$$

where $M$ is a constant. And since we can make $|O|>1 / 2$ on any compact subset $C$ of $G$,

$$
\int_{c}\left|T^{*} H\right| \leqq 2 M
$$

on an arbitrary compact subset $C$ of $G$, thereby showing that $T^{*} H$ is in $L_{1}(G)$.

6. An application. Let $G_{+}$be a closed semi-group of $G$ such that the interior of $G_{+}$is dense in $G_{+}, 1 \in G_{+}$, and the interior of $G_{+}$generates $G$. For such semi-groups $G_{+}$we propose to identify the multipliers of $L_{1}\left(G_{+}\right)=\left\{f \in L_{1}(G): f\right.$ vanishes off $\left.G_{+}\right\} . L_{1}\left(G_{+}\right)$is a closed subalgebra of $L_{1}(G)$. Let $\hat{G}$ be the dual of $G$ and let $A^{\prime}$ be the closed span of $\hat{G}$ in the norm of $L_{\infty}(G)$. Let $L_{1}(G) d x$ and $L_{1}\left(G_{+}\right) d x$ designate $L_{1}(G)$ and $L_{1}\left(G_{+}\right)$, respectively, considered as embedded (topologically) in $C_{0}(G)^{*}$.

Suppose $\mu \in M(G)$ has the property that for all $\mu_{f} \in L_{1}\left(G_{+}\right) d x$, $\mu * \mu_{f}=\mu_{o}$ where $\mu_{0} \in L_{1}\left(G_{+}\right) d x$. We will first show that $\mu$ must vanish off $G_{+}$.

Let $\left\{\mu_{\delta}\right\}$ be an approximate identity of $L_{1}(G) d x$ and let $\mu \in M(G)$. For any $h \in C_{0}(G)$, the set of continuous functions on $G$ vanishing at $\infty$, we have

$$
\mu * \mu_{\delta}(h)=\iint_{G} h(x y) \mu_{\delta}(d x) \mu(d y)=\iint_{G} h(x y) \mu_{\delta}(x) d x \mu(d y)
$$

and 


$$
\left|\mu * \mu_{\delta}(h)-\mu(h)\right|=\left|\iint_{G}(h(x y)-h(x)) \mu_{\delta}(x) d x \mu(d y)\right| .
$$

Since $y \rightarrow h^{v}\left(h^{v}(x)=h(x y)\right)$ is uniformly continuous on $G$, choose $V$ such that for $y \in V,\left\|h^{y}-h\right\|_{\infty}<\epsilon /\|\mu\|$. Then, taking $\mu_{\delta}$ with support $\left(\mu_{\delta}\right) \subset V$ we have

$$
\left|\iint_{G}(h(x y)-h(x))_{\mu_{\delta}}(x) d x \mu(d y)\right| \leqq\left\|h^{\nu}-h\right\|_{\infty}\|\mu\|<\epsilon .
$$

Therefore, pointwise on $C_{0}(G), \mu * \mu_{8} \rightarrow \mu$.

Suppose that support $(\mu) \cap\left(G-G_{+}\right) \neq \varnothing$, and let $N$ be a compact set with $|\mu(N)|>0$ and $N C \operatorname{support}(\mu) \cap G-G_{+}$. (This is possible, since $\mu$ is regular and $G_{+}$is closed.) Let $f \in C_{0}(G)$ be such that $f \equiv 1$ on a compact neighborhood $U$ of $N$ and $f \equiv 0$ off some open neighborhood $W$ of $N$. Let $V$ be a neighborhood of the identity satisfying $V \cdot N \subset U$. Choose $\left\{\mu_{\sigma}\right\}$ from an approximate identity of $L_{1}(G) d x$ with $\mu \in L_{1}\left(G_{+}\right) d x$. ( $G_{+}^{0}$ is dense in $G_{+}$and Haar measure is positive on open sets.) $\mu * \mu_{\sigma}(f) \rightarrow \mu(f) . \mu * \mu_{\sigma}$ is assumed an element of $L_{1}\left(G_{+}\right) d x$, hence is supported by $G_{+}$. Choosing $\mu_{0} \in\left\{\mu_{\sigma}\right\}$ such that support $\left(\mu_{0}\right) \subset V$, then

$$
\left|\mu * \mu_{0}(f)\right| \geqq\left|\int_{G \cap_{V N}} 1 \mu(d y)\right| \geqq|\mu(N)|>0
$$

in contradiction to the fact that support $\left(\mu * \mu_{0}\right) \subset G_{+}$. Hence any measure on $G$ which is a multiplier of $L_{1}\left(G_{+}\right)$must vanish off $G_{+}$.

Finally, we establish that such measures are precisely the multipliers of $L_{1}\left(G_{+}\right) \cdot \Delta\left(L_{1}(G)\right) \subset \Delta\left(L_{1}\left(G_{+}\right)\right), \Delta\left(L_{1}(G)\right)$ is the Silov boundary of $L_{1}\left(G_{+}\right)$in $\Delta\left(L_{1}\left(G_{+}\right)\right)$, and the topologies (relative and original) agree on $\Delta\left(L_{1}(G)\right)$. See [2]. Constructing $L_{1}\left(G_{+}\right)^{\prime \prime}$ using functionals in the closed span of the Silov boundary $\Delta\left(L_{1}(G)\right)$ in $\Delta\left(L_{1}\left(G_{+}\right)\right)$, we may still reason that $L_{1}\left(G_{+}\right)^{m} C C_{0}\left(G^{*}\right)^{*}=L_{1}(G)^{\prime \prime}$. Consequently, since any multiplier of $L_{1}\left(G_{+}\right)$must be continuous on $\Delta\left(L_{1}(G)\right)$, we can apply Eberlein's theorem to conclude that every multiplier of $L_{1}\left(G_{+}\right)$is in $M(G)$ and by the preceding paragraph must vanish off $G_{+}$.

THEOREM. The multipliers of $L_{1}\left(G_{+}\right)$are those measures in $M(G)$ with support contained in $G_{+}$.

\section{REFERENCES}

1. R. Arens, Operations induced on function classes, Monatsh. Math. 55 (1951), 1-19.

2. R. Arens and I. M. Singer, Generalized analytic functions, Trans. Amer. Math. Soc. 81 (1956), 379-393. 
3. F. T. Birtel, Banach algebras of multipliers, Duke Math. J. 28 (1961), 203-212.

4. - Isomorphisms and isometric multipliers, Proc. Amer. Math. Soc. 13 (1962), 204-210.

5. W. F. Eberlein, Characterizations of Fourier-Stieltjes transforms, Duke Math. J. 22 (1955), 465-468. $51-67$.

6. I. Glicksberg, Convolution semi-groups of measures, Pacific J. Math. 9 (1959),

7. L. H. Loomis, An introduction to abstract harmonic analysis, Van Nostrand, New York, 1953.

UNiversity of Notre Dame AND

YALE UNIVERSITY

\section{A GENERAL THEORY OF $k$-PLACE STROKE FUNCTIONS IN 2-VALUED LOGIC}

ATWELL R. TURQUETTE

Let $O$ be a 2 -valued operator which may be applied to $k(k \geqq 1)$ statements $P_{1}, \cdots, P_{k}$ to generate a new statement $O\left(P_{1}, \cdots, P_{k}\right)$. Let $o\left(p_{1}, \cdots, p_{k}\right)$ denote the truth-value function associated with $O\left(P_{1}, \cdots, P_{k}\right)$. We will assume that $o\left(p_{1}, \cdots, p_{k}\right)$ is defined by the standard truth table which is associated with the statement $O\left(P_{1}, \cdots, P_{k}\right)$ when 1 denotes "true" and 2 denotes "false." $A$ set of operators $\left\{O_{1}, \cdots, O_{t}\right\}$ will be called functionally complete if a statement calculus based on $O_{1}, \cdots, O_{t}$ is functionally complete. If the set $\{O\}$ is functionally complete, then $O$ will be called $a k$-place stroke and $O\left(P_{1}, \cdots, P_{k}\right)$ will be called $a k$-place stroke function. If $k=2$, there are exactly two $k$-place stroke functions; namely, the well-known $\uparrow\left(P_{1}, P_{2}\right)$ and $\downarrow\left(P_{1}, P_{2}\right)$ which are such that $\uparrow\left(p_{1}, p_{2}\right)$ $=3-\max \left(p_{1}, p_{2}\right)$ and $\downarrow\left(p_{1}, p_{2}\right)=3-\min \left(p_{1}, p_{2}\right)$. The purpose of this paper is to give a general method for constructing and calculating the number of $k$-place stroke functions for any given $k(1,2, \cdots, N)$. If $k=1$, then the set of $k$-place stroke functions is null. Hence, the present problem of generating $k$-place stroke functions becomes interesting only when $k \geqq 3$.

If a standard truth table is constructed for $O\left(P_{1}, \cdots, P_{k}\right)$, it will contain $2^{k}$ rows, and the $i$ th row will correspond to the $i$ th term in the lexicographical ordering of the sets of truth values $\left[v_{1}, \cdots, v_{k}\right]$ which are assigned to the arguments $P_{1}, \cdots, P_{k}$. Let $X_{i}$ denote the truth value of $O\left(P_{1}, \cdots, P_{k}\right)$ in the $i$ th row of a standard truth table for $O\left(P_{1}, \cdots, P_{k}\right)$. The truth values of $O\left(P_{1}, \cdots, P_{k}\right)$ which appear

Received by the editors August 8, 1961. 